\title{
Cross-cultural comparison of the sleep- disordered breathing prevalence among Americans and Japanese
}

\author{
K. Yamagishi*,\#, T. Ohira ", H. Nakano ${ }^{+}$, S.J. Bielinski ${ }^{\S}$, S. Sakurai ${ }^{\dagger}$, H. Imanoø, \\ M. Kiyama**, A. Kitamura**, S. Sato ${ }^{\# \#, ~ M . ~ K o n i s h i ' ~}{ }^{\dagger * *}$, E. Shahar" ${ }^{\star \Uparrow}$, A.R. Folsom", \\ H. Iso" and T. Tanigawa ${ }^{f}$
}

ABSTRACT: The aim of the present study was to compare the prevalence of sleep-disordered breathing among Hispanic and white Americans and Japanese.

A 1-night sleep study using a single-channel airflow monitor was performed on 211 Hispanics and 246 Whites from the Minnesota field centre (St Paul, MN, USA) of the Multi-Ethnic Study of Atherosclerosis (MESA), and 978 Japanese from three community-based cohorts of the Circulatory Risk in Communities Study (CIRCS) in Japan. The respiratory disturbance index and sleep-disordered breathing, defined as a respiratory disturbance index of $\geqslant 15$ events $\cdot \mathrm{h}^{-1}$, were estimated.

The prevalence of sleep-disordered breathing was higher in males $(34.2 \%)$ than females (14.7\%), and among Hispanics (36.5\%) and Whites (33.3\%) than among Japanese (18.4\%), corresponding to differences in body mass index. Within body mass index strata, the race difference in sleep-disordered breathing was attenuated. This was also true when body mass index was adjusted for instead of stratification. The strong association between body mass index and sleep-disordered breathing was similar in Japanese and Americans.

The prevalence of sleep-disordered breathing was lower among Japanese than among Americans. However, the association of body mass index with sleep-disordered breathing was strong, and similar among the race/ethnic groups studied. The majority of the race/ethnic difference in sleep-disordered breathing prevalence was explained by a difference in body mass index distribution.

KEYWORDS: Cross-sectional study, epidemiology, prevalence, sleep apnoea

leep-disordered breathing (SDB) is a condition of repetitive episodes of decreased or arrested respiratory airflow during sleep. In the USA, approximately one in five adults has at least mild obstructive sleep apnoea (OSA), a major category of SDB, and one in 15 adults has OSA of moderate or worse severity [1]. More than $85 \%$ of people with OSA who might benefit from treatment remain undiagnosed [1]. However, these prevalence estimates are confined to Whites since data in other races/ethnicities are scarce. Furthermore, lifestyle may also affect the prevalence of SDB, but data on cross-cultural differences in aetiology of SDB are scant.

A paradox has been suggested for Asians; compared with Whites, Asians are less obese, but may have an equivalent or even greater prevalence or severity of SDB [2, 3]. It is hypothesised that this is due to a different craniofacial profile compared with Whites $[3,4]$. However, this hypothesis is highly speculative since available prevalence data from Asia derive largely from hospital-based studies. The prevalence of SDB among Asians in community settings has been rarely reported [5-7] due to the difficulty of carrying out full polysomnography in such settings. In these contexts, the aim was to compare the prevalence of SDB among community-based populations of Hispanics and Whites in the USA, and Japanese living in Japan, using a portable sleep-monitoring device. Specifically, the focus was on the impact of body mass index (BMI), the most important risk factor for
AFFILIATIONS

*Dept of Public Health Medicine, Graduate School of Comprehensive Human Sciences and Institute of Community Medicine, University of Tsukuba, Tsukuba,

"Public Health, Dept of Social and Environmental Medicine, Osaka University Graduate School of Medicine, Suita,

+Dept of Pulmonology, Fukuoka National Hospital, Fukuoka, ${ }^{f}$ Dept of Public Health, Ehime University Graduate School of Medicine, Toon,

**Osaka Medical Center for Health Science and Promotion, Osaka, and ${ }^{\# \# C h i b a ~ P r e f e c t u r a l ~ I n s t i t u t e ~ o f ~}$ Public Health, Chiba, Japan. \#Division of Epidemiology and Community Health, School of Public Health, University of Minnesota, Minneapolis, MN,

${ }^{\text {s}}$ Dept of Health Sciences Research, Mayo Clinic, Rochester, MN, and

"Division of Epidemiology and Biostatistics, Mel and Enid Zuckerman College of Public Health, University of Arizona, Tucson, AZ, USA.

\section{CORRESPONDENCE}

T. Tanigawa, Dept of Public Health, Ehime University Graduate School of Medicine, Shitsukawa, Toon, Ehime 791-0295, Japan E-mail: Tt9178@aol.com

Received:

July 252009

Accepted after revision: Jan 182010 First published online: Jan 282010 
$\mathrm{SDB}$, on the race-specific prevalence of SDB. The a priori hypotheses were that: 1) the prevalence of SDB is higher among Hispanics and Whites than among Japanese, corresponding to higher BMIs, but 2) Japanese are more influenced by elevated BMI than Americans, such that the BMI-SDB association is stronger among Japanese than among Americans.

\section{METHODS}

\section{Study population}

Hispanic and white participants of the Minnesota field centre (St Paul, MN, USA) of the Multi-Ethnic Study of Atherosclerosis (MESA) and Japanese participants of the Akita-Osaka study and the Kyowa study, which constitute the Circulatory Risk in Communities Study (CIRCS), were included. In 2000-2002, the MESA recruited and assessed the cardiovascular risk factors of 6,814 males and females aged 4584 yrs who were free of clinically apparent cardiovascular disease at the time and lived in six US communities, including St Paul (MN, USA) [8]. They were followed by clinic visits every 2 yrs. In 2007, the MESA participants of the Minnesota field centre were recruited, who participated in the 4th MESA follow-up examination (2005-2007), were aged 50-74 yrs and living in the St Paul metropolitan area, and whose race/ ethnicity was Hispanic or white. The participants underwent a 1-night sleep study using a single-channel airflow monitor. Of 720 eligible participants, 464 provided informed consent and underwent the sleep study, 457 successfully.

The CIRCS is a dynamic Japanese community cohort consisting of five communities in Japan: Ikawa and Ishizawa in Akita, Yao in Osaka, Noichi in Kochi, and Kyowa in Ibaraki [9]. Annual cardiovascular check-ups were provided by the local governments. All residents aged $\geqslant 30$ yrs in each community were eligible to participate. During the 2006-2008 visits, participants at check-ups in Ikawa, Yao and Kyowa were asked to undergo the sleep study, using the same methods and devices as for the MESA. Of 6,233 people aged 50-74 yrs who participated in the check-ups, 978 randomly selected participants provided informed consent and completed the study successfully. Since $\sim 60 \%$ of the CIRCS participants were female (which is the case for most Japanese community health surveys), an annual recruiting plan was settled on in order to oversample male participants and be comparable with the MESA (table 3 of online supplementary material). Informed consent was obtained as approved by the local institutional review committees for the MESA, and those at the University of Tsukuba (Tsukuba, Japan), Osaka University (Suita, Japan) and Osaka Medical Center for Health Science and Promotion (Osaka, Japan) for the CIRCS.

\section{Measurement}

A single-channel airflow monitor, SOMNIE (NGK Spark Plug Co., Nagoya, Japan), was used to estimate the respiratory disturbance index (RDI). Details of this device have been published elsewhere [10]. Briefly, the SOMNIE detects nasal and oral breathing through a polyvinylidene fluoride film sensor, converts the airflow signals into digital data at a sampling frequency of $10 \mathrm{~Hz}$ and stores the data for $24 \mathrm{~h}$ until downloaded. The RDI was automatically derived from spectral analysis of the amplitude of airflow waves using Flow.exe software (Institute of Sleep Health Promotion, Tokyo, Japan)
[11]. Since the data were automatically processed, no interscorer variability existed in the present study. Examination (device recording) time was used instead of sleep time as the denominator for the calculation of RDI, since the exclusion of subjective awake time from the recording time in the sleep log proved useless in a validation study of this portable monitor [10]. Although the RDI is underestimated by this method, it was not affected very much in previous studies [10], partly due to the fact that the awake RDI is significantly higher in SDB subjects than in non-SDB subjects [11]. The repeatability $(\mathrm{r}=0.92$ between two 1-night tests) and validity (sensitivity of $89 \%$ and specificity of $96 \%$ for detection of an apnoea/ hypopnoea index $(\mathrm{AHI})$ of $\geqslant 30$ events $\cdot \mathrm{h}^{-1}$ using polysomnography as the gold standard) of RDIs derived from this device were fairly good [10], although the RDIs from the device tended to be higher than AHIs from full polysomnography (RDIs of 15.0, 11.2, 10.4 and 7.1 can be translated to AHIs of $12.5,9.0,8.2$ and 5.2, respectively). SDB was defined as an RDI of $\geqslant 15$ events $\cdot h^{-1}$.

Well-trained study staff explained and demonstrated to participants how to carry out the sleep study, and they performed the following measurements in the home (MESA) or at the health check-up site (CIRCS). Height was measured in stockinged feet and weight was measured wearing light clothing. BMI was calculated as weight (in kilograms) divided by the square of height (in metres). Smoking status, drinking status and the usual amount of alcohol consumption (in grams per day) were derived from a questionnaire.

\section{Statistical analysis}

In total, 211 Hispanics and 246 Whites from the MESA and 978 Japanese from the CIRCS were included in the present study. Differences in baseline characteristics among the race/ethnic groups were calculated using ANCOVA, Chi-squared tests or Kruskal-Wallis rank tests. Odds ratios (ORs) and 95\% confidence intervals (CIs) for SDB in relation to race, age and BMI group were calculated using logistic regression models. Covariates included age (except for analyses stratified by age), race (except for analyses stratified by race), BMI (except for analyses stratified by BMI), daily alcohol consumption (continuous) and smoking status (three categories: current, exsmoker or non-smokers). Interaction of BMI with race/ ethnicity in relation to SDB was tested in the logistic regression models using a cross-product term with BMI (continuous) and race/ethnicity (dichotomous). SAS version 9.1.3 Service Pack 4 (SAS Institute, Inc., Cary, NC, USA) was used for the analyses. All probabilities for statistical tests were two-tailed, and a pvalue of $<0.05$ was regarded as significant.

As supplementary analyses, similar analyses were performed for questionnaire-based self-reported snoring and nocturnal apnoea in the whole MESA cohort attending visit $4(n=3,257$, further including African Americans but not Chinese Americans) and in the CIRCS participants ( $n=5,785$ at the 2000-2005 surveys) aged 50-74 yrs. Chinese Americans were excluded from the MESA because of the small sample size $(n=430)$ and the narrow distribution of BMI $(85 \%$ of Chinese Americans had a BMI ranging $17.5-27.4 \mathrm{~kg} \cdot \mathrm{m}^{-2}$ ). The proportions of "don't know if they snore or not" were lower among Japanese ( $7 \%$ for males and $15 \%$ for females) than Hispanics ( $21 \%$ for males and $28 \%$ for females), Blacks (24\% for males 
and $34 \%$ for females), Whites $(20 \%$ for males and $29 \%$ for females) and Chinese Americans (22\% for males and 32\% for females). Sex-specific scatter diagrams of RDI by age and BMI for the three ethnic groups are presented in the online supplementary material.

\section{RESULTS}

The age and sex distributions were similar across the race/ethnic groups (table 1). For males, the proportion of current drinkers was greater among Whites and Japanese than among Hispanics. For females, this proportion was largest among Whites, followed by Hispanics and Japanese. The proportion of current smokers was larger among Japanese males than American males, and was larger among white females than Hispanic and Japanese females. The median RDI was 11.2 events $\cdot h^{-1}$ among Whites, 10.4 events $\cdot \mathrm{h}^{-1}$ among Hispanics and 7.1 events $\cdot \mathrm{h}^{-1}$ among Japanese. The prevalence of SDB was higher among Hispanics $(36.5 \% ; n=77)$ and Whites $(33.3 \% ; n=82)$ than among Japanese $(18.4 \% ; n=180)$. Correspondingly, mean BMI was higher among Hispanics $\left(31.5 \mathrm{~kg} \cdot \mathrm{m}^{-2}\right)$ and Whites $\left(29.0 \mathrm{~kg} \cdot \mathrm{m}^{-2}\right)$ than among Japanese $\left(23.5 \mathrm{~kg} \cdot \mathrm{m}^{-2}\right)$. Median RDIs were higher in males $\left(10.0\right.$ events $\left.\cdot h^{-1}\right)$ than in females $\left(7.0\right.$ events $\left.\cdot h^{-1}\right)$, and the prevalence of SDB was approximately twice as high in males as females (34.2 versus 14.7\%). The race/ethnic differences were similarly observed between males and females.

The association between age and prevalent SDB was weak among Whites compared with Hispanics and Japanese (table 2) for both males and females (fig. E1 of online supplementary material). Conversely, the prevalence of SDB was similar in BMI strata across the race/ethnic groups (table 2), although the scatter diagram of RDI and BMI showed that the RDI was lower in high BMI strata among Japanese females than among Hispanic and white females (fig. E2 of online supplementary material). For example, the SDB prevalence for those with a BMI of $22.5-24.9 \mathrm{~kg} \cdot \mathrm{m}^{-2}$ was $17.2 \%$ among Japanese, $18.2 \%$ among Whites and $20.0 \%$ for Hispanics (p-value for overall difference 0.95). Similarly, the SDB prevalence for those with a BMI of $\geqslant 30.0 \mathrm{~kg} \cdot \mathrm{m}^{-2}$ was $35.3 \%$ among Japanese, $43.8 \%$ among Whites and $40.0 \%$ among Hispanics (p-value for overall difference 0.67 ) (table 2). The multivariable-adjusted ORs (95\% CI) for SDB for those with a BMI of $\geqslant 30.0 \mathrm{~kg} \cdot \mathrm{m}^{-2}$ compared with a BMI of 22.5 $24.9 \mathrm{~kg} \cdot \mathrm{m}^{-2}$ were also similar between the Japanese and MESA participants, 4.15 (1.86-9.25) and 3.49 (1.53-7.92), respectively. It should be noted that the prevalence of SDB for those with a BMI of $30.0-32.4 \mathrm{~kg} \cdot \mathrm{m}^{-2}$ was $42.1 \%(\mathrm{n}=16)$ for Whites, $32.6 \%$ $(n=14)$ for Hispanics and $40.9 \%(n=9)$ for Japanese, albeit with small sample sizes. When BMI was expressed as a continuous variable, the multivariable-adjusted OR (95\% CI) per $5 \mathrm{~kg} \cdot \mathrm{m}^{-2}$ increment was $1.82(1.39-2.38)$ in Japanese and 1.42 (1.17-1.72) in Hispanic and Whites pooled. There was no significant interaction of $\mathrm{BMI}$ with race/ethnicity in relation to SDB $(\mathrm{p}=0.11)$

The multivariable-adjusted ORs (95\% CI) for SDB were 2.82 (1.98-4.02) for Hispanics compared with Japanese and 2.52 (1.81-3.51) for Whites compared with Japanese. As expected, further adjustment for BMI attenuated the association (1.50 (0.98-2.31) for Hispanics and 1.62 (1.11-2.36) for Whites (data not shown)). Race did not greatly confound the association of $\mathrm{BMI}$ with SDB; in the total sample, the multivariable-adjusted OR $(95 \% \mathrm{CI})$ for SDB per $5 \mathrm{~kg} \cdot \mathrm{m}^{-2}$ increment of SDB was 1.70 (1.50-1.93) before adjustment for race and 1.51 (1.29-1.77) after adjustment for race.

Similarly, the prevalences of questionnaire-based snoring and apnoea outcomes (tables E1 and E2 of online supplementary material) were similar among most race/ethnic groups within each BMI stratum. The exception was that Japanese females exhibited the lowest prevalence of sleep apnoea in almost all BMI strata.

TABLE 1 Race/ethnicity-specific characteristics of sleep study participants ${ }^{\#}$

\begin{tabular}{|c|c|c|c|c|c|c|c|c|}
\hline & Subjects $\mathrm{n}$ & Age yrs & Males \% & RDI events $\cdot h^{-1}$ & SDB $\%$ & BMI $\mathbf{k g} \cdot \mathbf{m}^{-2}$ & Drinkers $^{+} \%$ & Smokers $^{+} \%$ \\
\hline \multicolumn{9}{|l|}{ Total } \\
\hline White & 246 & 62.0 & 44 & 11.2 & 33.3 & 29.0 & 66 & 17 \\
\hline Japanese & 978 & 63.0 & 46 & 7.1 & 18.4 & 23.5 & 44 & 16 \\
\hline$p$-value $e^{\S}$ & & 0.02 & 0.70 & $<0.001$ & $<0.001$ & $<0.001$ & $<0.001$ & 0.03 \\
\hline White & 108 & 62.1 & & 15.1 & 50.9 & 28.9 & 71 & 18 \\
\hline Japanese & 446 & 63.5 & & 8.3 & 27.1 & 23.8 & 75 & 30 \\
\hline$p$-value $e^{\S}$ & & 0.01 & & $<0.001$ & $<0.001$ & $<0.001$ & $<0.001$ & $<0.001$ \\
\hline \multicolumn{9}{|l|}{ Females } \\
\hline Hispanic & 110 & 62.0 & & 9.5 & 26.4 & 32.2 & 36 & 6 \\
\hline White & 138 & 61.9 & & 9.3 & 19.6 & 29.0 & 62 & 17 \\
\hline
\end{tabular}

Data are presented as means, with RDI as medians, unless otherwise indicated. RDI: respiratory disturbance index; SDB: sleep-disordered breathing; BMI: body mass index. " : Minnesota Multi-Ethnic Study of Atherosclerosis (MESA; Hispanics and Whites) and Circulatory Risk in Communities Study (CIRCS; Japanese) subjects aged 50-74 yrs; ${ }^{\bullet}$ : defined by RDI of $\geqslant 15$ events $\cdot h^{-1} ;{ }^{+}$: current; ${ }^{\S}$ : for overall difference 


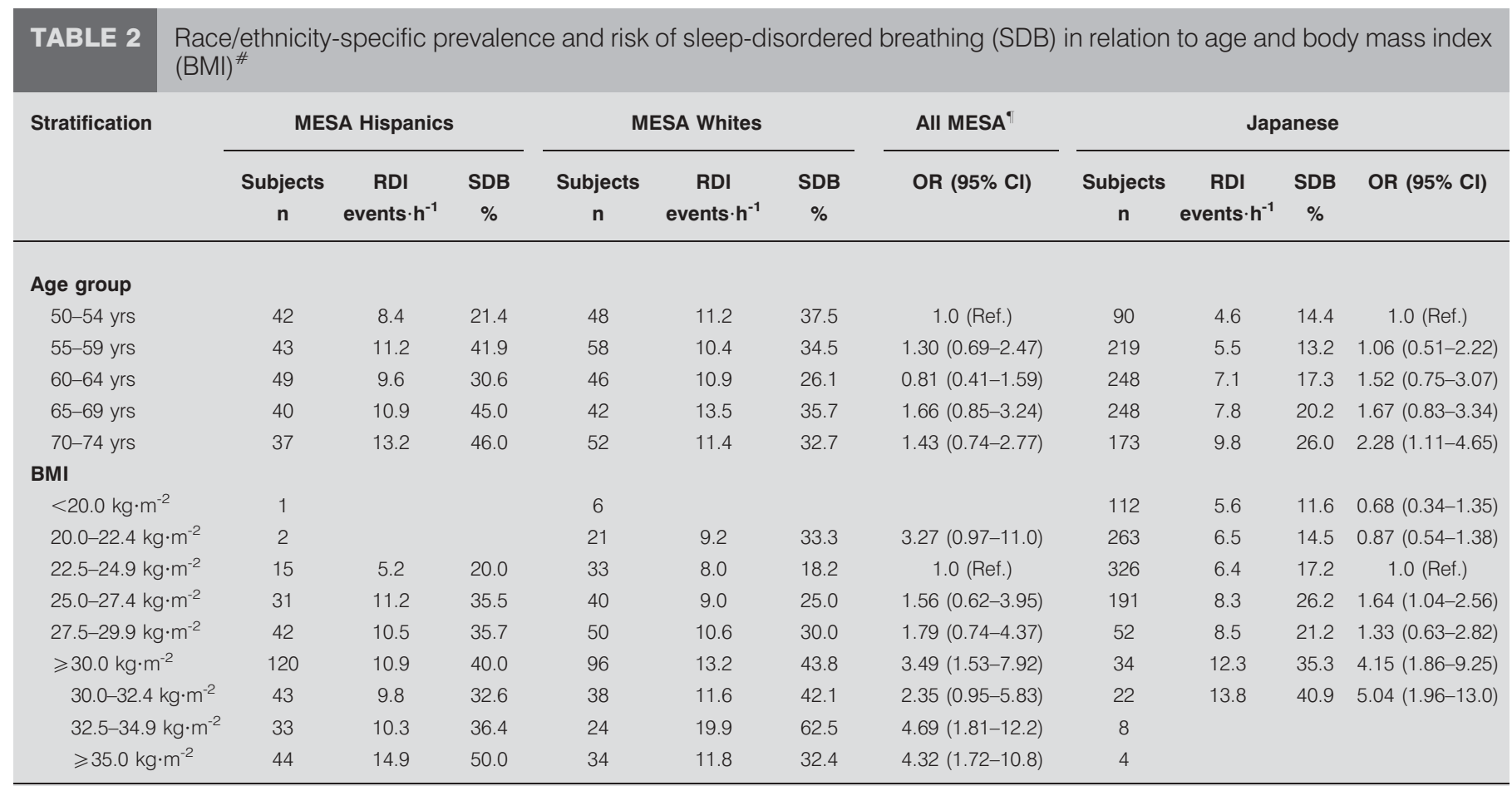

Data are presented as medians, unless otherwise indicated. Odds ratios (ORs) were adjusted for age (except in age stratification), sex, amount of daily alcohol consumption, smoking status and BMI (except in those stratified by BMI). Further adjustment for race was carried out for the Minnesota Multi-Ethnic Study of Atherosclerosis (MESA). Persons with missing alcohol or smoking status $(n=6)$ were eliminated. RDI: respiratory disturbance index; Cl: confidence interval; Ref. reference group. " : in MESA (Hispanics and Whites) and Circulatory Risk in Communities Study (CIRCS; Japanese) subjects; ": Whites and Hispanics pooled.

\section{DISCUSSION}

As hypothesised, the prevalence of SDB was lower among Japanese than in the St Paul MESA sample. However, contrary to the second hypothesis, that Japanese are more affected by elevated BMI than Hispanics and Whites, it was found that the association of BMI with SDB was strong and homogeneous between the race/ethnic groups, i.e. there was no effect modification on the multiplicative scale. Furthermore, the difference in SDB prevalence between the Japanese and MESA samples was largely explained by a difference in BMI distribution, since the adjustment for BMI eliminated the race/ethnic differences in SDB prevalence. The prevalence of $\mathrm{SDB}$, in both non-obese (e.g. BMI of $22.5-24.9 \mathrm{~kg} \cdot \mathrm{m}^{-2}$ ) and obese (BMI of $\geqslant 30.0 \mathrm{~kg} \cdot \mathrm{m}^{-2}$ ) strata, did not differ significantly among Hispanics, Whites and Japanese. Supplemental analyses of questionnaire-based snoring and apnoea agreed with these results, although females, especially Japanese females, showed a generally lower prevalence of SDB in the same BMI stratum than males.

Our hypothesis was based on a previous report that male sleep apnoea patients of Far East Asian extraction (Chinese, Japanese and Korean), despite being non-obese, showed greater severity of RDI than did white males; this corresponded to decreased cranial base dimensions (i.e. shorter anterior cranial base and narrower cranial base fracture) [4]. However, posterior airway space and hyoid position, which were also associated with prevalent OSA among Asians in that study, were less abnormal in Asian males than white males [4]. A Japanese study reported that the cephalometric characteristics of
Japanese sleep apnoea patients were similar to those of white patients [12]. Craniofacial difference undoubtedly contributes to the development of SDB, but the racial/ethnic difference in the prevalence or severity of SDB seems to be difficult to explain solely on the basis of cephalometric profile. Another Japanese study showed that cephalometric profile interacted with $\mathrm{BMI}$ in relation to SDB, i.e. craniofacial profile (based on cranial base dimensions and hyoid position) was associated with the prevalence of SDB more among males with higher BMI than those with lower BMI [13].

Although the present results suggested that the prevalence of SDB correlated strongly with BMI across all race/ethnic groups, it should be noted that SDB is frequent even among non-obese people. Indeed, the prevalence of SDB was substantial (17-20\%) among those with a lean BMI of 22.5$24.9 \mathrm{~kg} \cdot \mathrm{m}^{-2}$ regardless of cultural setting. This means that paying attention only to typical symptoms, characterised as Pickwickian (often described as extremely obese always sleepy middle-aged males) may overlook sleep apnoea in non-obese patients [14].

The present study is the first to compare the prevalence of SDB directly between Hispanics, Whites and Japanese in different community-based settings using identical procedures. Usage of a portable one-channel flow sensor device, instead of polysomnography, has both advantages and limitations. The reliability and validity of the device was fairly good and quite comparable with full polysomnography [10]. The traditional thermal sensors have been reported to be likely to overlook 
hypopnoea events, and the polyvinylidene fluoride film sensor used here has excellent ability at detecting hypopnoea [15]. This portable device afforded a simple procedure for a 1night test in the participant's own home, which might have reduced resistance to participation in the study. Conversely, the RDIs obtained were not identical to AHIs from polysomnography, and oxygen desaturation was not measured; this should be considered when comparing the present prevalences of SDB with those from elsewhere. We are aware that the present RDIs were overestimated compared with AHIs from polysomnography, as stated in the Methods section. Moreover, the median RDIs from the present study were substantially higher than the AHIs of landmark studies, such as the Wisconsin Sleep Cohort Study [16] and the Sleep Heart Health Study [17].

Other study limitations warrant discussion. First, although the participants were from community-dwelling general populations, bias related to self-selection is possible, i.e. people with sleep problems may have been likely to participate in the present study. However, this issue applies to every sleep study, and, again, the present study may be less affected by this than studies using the more burdensome polysomnography. Indeed, baseline characteristics did not differ greatly between participants and nonparticipants in both the MESA and the CIRCS, although participants were somewhat younger than nonparticipants in the CIRCS (table E3 of online supplementary material). Secondly, since the distribution of BMI was quite different between Japanese and Americans, those with a BMI of $\geqslant 30 \mathrm{~kg} \cdot \mathrm{m}^{-2}$ were pooled into one stratum, which may leave residual confounding by BMI in that stratum. However, the prevalence of SDB did not differ greatly across race/ethnicities in the stratum of $30.0-32.5 \mathrm{~kg} \cdot \mathrm{m}^{-2}$, and so this may not greatly affect the results. Thirdly, although a large difference in SDB prevalence between males and females was observed, it was not possible to conduct sex-specific analyses due to the small sample sizes. However, the patterns were similar when examined sex-specifically (figs. E1 and E2 of online supplementary material) and by the supplementary sexspecific analyses of self-reported snoring and nocturnal apnoea. Finally, this is a cross-sectional study, and so it cannot be proven that the association of BMI with SDB is causal.

In conclusion, the prevalence of SDB was lower in a Japanese population-based cohort than in the St Paul MESA sample. However, the strong association of BMI with SDB was similar between the ethnic groups studied, and most of the difference in SDB prevalence between these Japanese and Americans was explained by a difference in BMI distribution.

\section{SUPPORT STATEMENT}

The Multi-Ethnic Study of Atherosclerosis (MESA) was funded by National Heart, Lung, and Blood Institute (Bethesda, MD, USA) grant (contracts N01-HC-95159 to N01-HC-95165 and N01-HC-95169). The Circulatory Risk in Communities Study (CIRCS) was partly funded by Grant-in-Aid for Scientific Research (17659184, 18390194 and 18659179) from the Ministry of Education, Culture, Sports, Science and Technology (Tokyo, Japan). This ancillary study was also supported by the Pfizer Health Research Foundation (Tokyo, Japan), the Osaka Medical Research Foundation for Incurable Diseases (Osaka, Japan), the Japan Cardiovascular Research Foundation (Suita, Japan) and the ResMed Foundation (La Jolla, CA, USA).

\section{STATEMENT OF INTEREST}

A statement of interest for this study can be found at www.erj. ersjournals.com $/ \mathrm{misc} /$ statements.dtl

\section{ACKNOWLEDGEMENTS}

The authors thank the other investigators, staff and participants of the Multi-Ethnic Study of Atherosclerosis (MESA) and Circulatory Risk in Communities Study (CIRCS) for their valuable contributions. We acknowledge I. Muraki, M. Nagayoshi and K. Maruyama (Osaka University, Suita, Japan) who contributed to the CIRCS sleep data collection.

The CIRCS is a collaborative study managed by the Osaka Medical Center for Health Science and Promotion (Osaka, Japan), University of Tsukuba (Tsukuba, Japan), Osaka University and Ehime University (Toon, Japan).

The CIRCS investigators who contributed to the present study are as follows: M. Konishi ${ }^{\dagger}$, Y. Ishikawa, M. Nakamura, A. Kitamura, M. Kiyama, T. Okada, K. Maeda, M. Ido, M. Nakamura, K. Kamei, T. Shimamoto, M. Iida, and Yoshio Komachi (Osaka Medical Center for Health Science and Promotion); S. Sato (Chiba Prefectural Institute of Public Health, Chiba, Japan); K. Yamagishi, K. Kirii, M. Umesawa, C-L. Chei, K. Yokota, and M. Tabata (University of Tsukuba); H. Iso, T. Ohira, H. Imano, R. Cui, and S. Ikehara (Osaka University); T. Tanigawa, I. Saito, K. Okada, and S. Sakurai (Ehime University); M. Yao (Ranryoen Hospital, Ibaraki, Japan); and A. Ikeda, and H. Noda (Harvard School of Public Health, Boston, MA, USA).

A full list of participating investigators and institutions can be found at www.mesa-nhlbi.org for the MESA.

\section{REFERENCES}

1 Somers VK, White DP, Amin R, et al. Sleep apnea and cardiovascular disease. An American Heart Association/American College of Cardiology Foundation Scientific Statement from the American Heart Association Council for High Blood Pressure Research Professional Education Committee, Council on Clinical Cardiology, Stroke Council, and Council on Cardiovascular Nursing, In Collaboration with the National Heart, Lung, and Blood Institute National Center on Sleep Disorders Research (National Institutes of Health). Circulation 2008; 118: 1080-1111.

2 Ong KC, Clerk AA. Comparison of the severity of sleepdisordered breathing in Asian and Caucasian patients seen at a sleep disorders center. Respir Med 1998; 92: 843-848.

3 Li KK, Powell NB, Kushida C, et al. A comparison of Asian and white patients with obstructive sleep apnea syndrome. Laryngoscope 1999; 109: 1937-1940.

4 Li KK, Kushida C, Powell NB, et al. Obstructive sleep apnea syndrome: a comparison between Far-East Asian and white men. Laryngoscope 2000; 110: 1689-1693.

5 Ip MSM, Lam B, Tang LCH, et al. A community study of sleepdisordered breathing in middle-aged Chinese women in Hong Kong: prevalence and gender differences. Chest 2004; 125: 127-134.

6 Ip MSM, Lam B, Lauder IJ, et al. A community study of sleepdisordered breathing in middle-aged Chinese men in Hong Kong. Chest 2001; 119: 62-69.

7 Kim J, In K, Kim J, et al. Prevalence of sleep-disordered breathing in middle-aged Korean men and women. Am J Respir Crit Care Med 2004; 170: 1108-1113.

8 Bild DE, Bluemke DA, Burke GL, et al. Multi-Ethnic Study of Atherosclerosis: objectives and design. Am J Epidemiol 2002; 156: 871-881.

9 Iso H, Sato S, Kitamura A, et al. Metabolic syndrome and the risk of ischemic heart disease and stroke among Japanese men and women. Stroke 2007; 38: 1744-1751. 
10 Nakano H, Tanigawa T, Ohnishi Y, et al. Validation of a singlechannel airflow monitor for screening of sleep-disordered breathing. Eur Respir J 2008; 32: 1060-1067.

11 Nakano H, Tanigawa T, Furukawa T, et al. Automatic detection of sleep-disordered breathing from a single-channel airflow record. Eur Respir J 2007; 29: 728-736.

12 Sakakibara H, Tong M, Matsushita K, et al. Cephalometric abnormalities in non-obese and obese patients with obstructive sleep apnoea. Eur Respir J 1999; 13: 403-410.

13 Yao M, Tachibana N, Okura M, et al. Relationships of craniofacial morphology and body mass index with sleep-disordered breathing in Japanese men. Laryngoscope 2004; 114: 1838-1842.
14 American Academy of Sleep Medicine, The International Classification of Sleep Disorders. Westchester, American Academy of Sleep Medicine, 2005.

15 Berry RB, Koch GL, Trautz S, et al. Comparison of respiratory event detection by a polyvinylidene fluoride film airflow sensor and a pneumotachograph in sleep apnea patients. Chest 2005; 128: 1331-1338.

16 Young T, Palta M, Dempsey J, et al. The occurrence of sleepdisordered breathing among middle-aged adults. N Engl J Med 1993; 328: 1230-1235.

17 Young T, Shahar E, Nieto FJ, et al. Predictors of sleep-disordered breathing in community-dwelling adults: the Sleep Heart Health Study. Arch Intern Med 2002; 162: 893-900. 